\title{
Electrons and Phonons in High Temperature Superconductors
}

\author{
Anu Singh, ${ }^{1}$ Hempal Singh, ${ }^{1}$ Vinod Ashokan, ${ }^{2}$ and B. D. Indu ${ }^{1}$ \\ ${ }^{1}$ Department of Physics, Indian Institute of Technology, Roorkee 247667, Uttarakhand, India \\ ${ }^{2}$ Department of Physics, Texas A\&M University, Education City, Doha 23874, Qatar
}

Correspondence should be addressed to B. D. Indu; drbdindu@gmail.com

Received 22 November 2012; Accepted 31 December 2012

Academic Editor: Zhiqiang Mao

Copyright (C) 2013 Anu Singh et al. This is an open access article distributed under the Creative Commons Attribution License, which permits unrestricted use, distribution, and reproduction in any medium, provided the original work is properly cited.

\begin{abstract}
The defect-induced anharmonic phonon-electron problem in high-temperature superconductors has been investigated with the help of double time thermodynamic electron and phonon Green's function theory using a comprehensive Hamiltonian which includes the contribution due to unperturbed electrons and phonons, anharmonic phonons, impurities, and interactions of electrons and phonons. This formulation enables one to resolve the problem of electronic heat transport and equilibrium phenomenon in high-temperature superconductors in an amicable way. The problem of electronic heat capacity and electronphonon problem has been taken up with special reference to the anharmonicity, defect concentration electron-phonon coupling, and temperature dependence.
\end{abstract}

\section{Introduction}

With the remarkable discovery of high-temperature superconductivity (HTSC) in the Ba-La-Cu-O system with $T_{c} \sim$ $30 \mathrm{~K}$ by Bednorz and Mullers there begins a new exciting era in condensed matter physics because of their variety of applications in science and technology. The pairing mechanism in high temperature superconductors (HTS), however, being an unresolved problem, there are large number of experimental evidences that the electron-phonon (e-p) interaction together with strong electronic correlations plays a decisive role in understanding the phenomenon of superconductivity [1]. In the literature, it is reported that e-p coupling plays a crucial role in determining the electron density of states (EDOS) and electronic heat capacity (EHC). The specific heat which can be determined from temperature dependence and the spectrum of electrons and phonons has always been a central one in view of its importance in understanding the lowtemperature phenomenon in solids. The total heat capacity of HTS is contributed by lattice heat capacity (LHC) and EHC [2]. The EHC $(\sim \gamma T)$ is only appreciable at low-temperatures and changes dramatically at the superconducting transition, whereas the phonon contribution dominates at room temperature and is generally undisturbed by the transition at $T_{c}$. The Sommerfeld constant $\gamma=\left(2 \pi k_{B}^{2} / 3\right) D\left(\epsilon_{F}\right)$ provides an important test for proposed theories [3-5], where $D\left(\epsilon_{F}\right)$ is the EDOS evaluated at Fermi energy $\epsilon_{F}$.

In the present work, the expressions for EDOS and EHC have been obtained with the help of many body Green's function theory which uses an almost complete Hamiltonian via quantum dynamics of electrons and phonons.

\section{The Hamiltonian and Green's Functions}

In order to formulate the problem with special reference to HTS, we consider an almost complete (without BCS type) Hamiltonian $[6,7]$ in the form:

$$
\begin{aligned}
H= & H_{e}+H_{e p}+H_{A}+H_{p}+H_{D} \\
= & \sum_{q}\left(\epsilon_{q \uparrow} b_{q \uparrow}^{*} b_{q \uparrow}+\epsilon_{q \downarrow} b_{q \downarrow}^{*} b_{q \downarrow}+\epsilon_{-q \uparrow} b_{-q \uparrow}^{*} b_{-q \uparrow}\right. \\
& \left.\quad+\epsilon_{-q \downarrow} b_{-q \downarrow}^{*} b_{-q \downarrow}\right) \\
& +\sum_{q k}\left(g_{k} b_{Q \uparrow}^{*} b_{q \uparrow}+g_{k}^{*} b_{q \uparrow}^{*} b_{Q \uparrow}+g_{k} b_{Q \downarrow}^{*} b_{q \downarrow}+g_{k}^{*} b_{q \downarrow}^{*} b_{Q \downarrow}\right) B_{k} \\
& +\sum_{s \geq 3} \sum_{k_{1} \cdots k_{s}} V_{s}\left(k_{1}, k_{2} \cdots k_{s}\right) A_{k_{1}} A_{k_{2}} \cdots A_{k_{s}}
\end{aligned}
$$




$$
\begin{aligned}
& +\sum_{k} \frac{\epsilon_{k}}{4}\left(A_{k}^{*} A_{k}+B_{k}^{*} B_{k}\right) \\
& +\sum_{k_{1}, k_{2}}\left[-C\left(k_{1}, k_{2}\right) B_{k_{1}} B_{k_{2}}+D\left(k_{1}, k_{2}\right) A_{k_{1}} A_{k_{2}}\right]
\end{aligned}
$$

where $H_{e}, H_{e p}, H_{A}, H_{p}$, and $H_{D}$ describe the contributions to the Hamiltonian coming from unperturbed electrons, electron-phonon coupling, anharmonicities, harmonic phonons and defects, respectively. $A_{k}, B_{k}$, and $b_{q}\left(b_{q}^{*}\right)$ are the phonon field, phonon momentum, and electron annihilation (creation) operators with spins up $(\uparrow)$ or down $(\downarrow)$, respectively. $\vec{Q}=\vec{k}+\vec{q} . V_{s}\left(k_{1}, k_{2} \cdots k_{s}\right)$ represents anharmonic coupling cofficients [8] and $C\left(k_{1}, k_{2}\right)$ and $D\left(k_{1}, k_{2}\right)$ are the mass and force constant change parameters [9], respectively.

Now we consider the evaluation of one electron Green's function:

$$
\begin{aligned}
G_{q, q^{\prime}}\left(t-t^{\prime}\right) & =\left\langle\left\langle b_{q \sigma}(t) ; b_{q^{\prime} \sigma^{\prime}}^{*}\left(t^{\prime}\right)\right\rangle\right\rangle \\
& =-i \theta\left(t-t^{\prime}\right)\left\langle\left[b_{q \sigma}(t), b_{q^{\prime} \sigma^{\prime}}^{*}\left(t^{\prime}\right)\right]\right\rangle
\end{aligned}
$$

via Hamiltonian (1) and adopting the quantum dynamical approach of electrons and phonons [10]. After some simplifications and Dyson equation approach, this can be obtained in the form:

$$
\begin{aligned}
G_{q, q^{\prime}}(\epsilon)= & \left(3 \epsilon_{q}+\epsilon_{q}^{c}\right)(2 \pi)^{-1} \\
& \times\left[\epsilon^{2}-\bar{\epsilon}_{q}^{2}+i\left(3 \epsilon_{q}+\epsilon_{q}^{c}\right) \Gamma_{q}(\epsilon)\right]^{-1} \delta_{q q^{\prime}} \delta_{\sigma \sigma^{\prime}} .
\end{aligned}
$$

with $\bar{\epsilon}_{q}^{2}=\widetilde{\epsilon}_{q}^{2}+\left(3 \epsilon_{q}+\epsilon_{q}^{c}\right) \Delta_{q}(\epsilon)$. In the above expressions $\widetilde{\epsilon}_{q}, \bar{\epsilon}_{q}$ and $\epsilon_{q}^{c}$ are renormalized mode, perturbed mode, and pairon energies, respectively. $\Delta_{q}(\epsilon)$ and $\Gamma_{q}(\epsilon)$ are the electron energy line shifts and widths, respectively [11]. The electron energy line width $\Gamma_{q}(\epsilon)$ has the form:

$$
\begin{aligned}
\Gamma_{q}(\epsilon)=\Gamma_{q}^{D}(\epsilon)+\Gamma_{q}^{3 A}(\epsilon)+\Gamma_{q}^{e p}(\epsilon), \\
\Gamma_{q}^{D}(\epsilon)=512 \pi \sum_{k, k_{1}}\left|D\left(k, k_{1}\right)\right|^{2} \\
\times\left[\xi(\epsilon) \epsilon_{k_{1}} N\left(\epsilon_{k c}\right) \delta\left(\epsilon^{2}-\widetilde{\epsilon}_{k_{1}}^{2}\right)+n_{k_{1}} \delta\left(\epsilon-\epsilon_{q c}\right)\right] \\
\times\left(\epsilon_{q c}\right)^{-2}, \\
\Gamma_{q}^{3 A}(\epsilon)=512 \pi \sum_{k, k_{1}, k_{2}}\left|V_{3}\left(k, k_{1}, k_{2}\right)\right|^{2} \\
\times\left[\xi(\epsilon) \epsilon_{k_{1}} \eta_{1} N\left(\epsilon_{k c}\right) A_{\alpha}+n_{k_{1}} n_{k_{2}} \delta\left(\epsilon-\epsilon_{q c}\right)\right] \\
\times\left(\epsilon_{q c}\right)^{-2}, \\
\Gamma_{q}^{e p}(\epsilon)=16 \pi \sum_{k}\left|g_{k}\right|^{2}\left[\xi(\epsilon) N\left(\epsilon_{k c}\right) \delta\left(\epsilon^{2}-\widetilde{\epsilon}_{k}^{2}\right) \Omega\right. \\
\left.+\widetilde{\Omega} \delta\left(\epsilon-\epsilon_{q c}\right)\right] .
\end{aligned}
$$

In the present work, we have taken the contribution of anharmonicities up to cubic order because the higher-order anharmonicities are appreciable at high temperatures.

\section{Electron Density of States}

Using Lehman's representation and Green's function formalism with some algebra, the EDOS can be obtained in the form:

$$
\begin{aligned}
D(\epsilon)= & (2 \pi)^{-1} \sum_{q}\left(3 \epsilon_{q}+\epsilon_{q}^{c}\right)^{2} \Gamma_{q}(\epsilon) \\
& \times\left[\left(\epsilon^{2}-\bar{\epsilon}_{q}^{2}\right)^{2}+\left(3 \epsilon_{q}+\epsilon_{q}^{c}\right)^{2} \Gamma_{q}^{2}(\epsilon)\right]^{-1} \delta_{q q^{\prime}} \delta_{\sigma \sigma^{\prime}} .
\end{aligned}
$$

For low values of $\Gamma_{q}(\epsilon), D(\epsilon)$ can be approximated via BreitWigner approximation. In the limiting case, when electron line width is very small but finite, $D(\epsilon)$ shows a steep maximum at $\epsilon=\bar{\epsilon}_{q}$ and density of state behaves as Lorentzian line shape function peaked at $\epsilon=\bar{\epsilon}_{q}$.

\section{The Electron Energy}

Using (5), one can get the energy of an electron in the form:

$$
E=E^{D}+E^{3 A}+E^{e p} .
$$

The different terms appeared in the above energy equation are

$$
\begin{aligned}
E^{D}= & 128 \sum_{k, k_{1}}\left|g_{k}\right|^{2}\left|D\left(k_{1}, k\right)\right|^{2} \\
\times & {\left[N\left(\epsilon_{k c}\right) \epsilon_{k_{1}} n\left(\widetilde{\epsilon}_{k_{1}}\right) D\left(\widetilde{\epsilon}_{k_{1}}, \widetilde{\epsilon}_{q}\right)\right.} \\
& \left.+2 n_{k_{1}} \widetilde{\epsilon}_{q c} n\left(\widetilde{\epsilon}_{q c}\right) D\left(\widetilde{\epsilon}_{q c}, \bar{\epsilon}_{q}\right)\right], \\
E^{3 A}= & 288 \sum_{k_{1}, k_{2}, k}\left|V_{3}\left(k_{1}, k_{2}, k\right)\right|^{2} \\
\times & {\left[\eta_{1} N\left(\epsilon_{k c}\right) D_{s}\left(\widetilde{\epsilon}_{ \pm \alpha}, \widetilde{\epsilon}_{q}\right)\right.} \\
& \left.+2 n_{k_{1}} n_{k_{2}} \widetilde{\epsilon}_{q c} n\left(\widetilde{\epsilon}_{q c}\right) D\left(\widetilde{\epsilon}_{q c}, \bar{\epsilon}_{q}\right)\right], \\
E^{e p}= & 4 \sum_{k}\left|g_{k}\right|^{2} \epsilon_{q c}^{2}\left[N\left(\epsilon_{k c}\right) n\left(\widetilde{\epsilon}_{k}\right) D\left(\widetilde{\epsilon}_{k}, \widetilde{\epsilon}_{q}\right) \Omega\right. \\
& \left.+2 \widetilde{\epsilon}_{q c} n\left(\widetilde{\epsilon}_{q c}\right) \widetilde{\Omega} D\left(\widetilde{\epsilon}_{q c}, \bar{\epsilon}_{q}\right)\right] .
\end{aligned}
$$




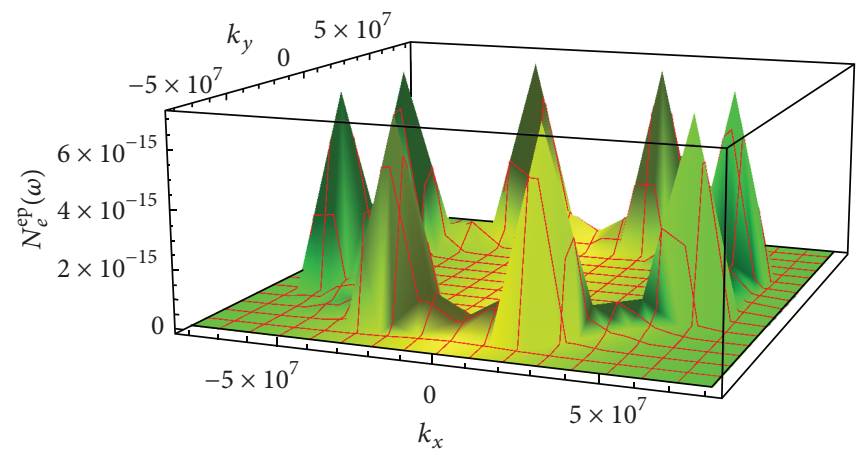

FIGURE 1: Electronic excitation due to electron-phonon interaction.

\section{The Electronic Heat Capacity}

We now readily obtain the expression for EHC in the following form:

$$
\begin{aligned}
& C_{e l}(T)=C_{e l}^{D}(T)+C_{e l}^{3 A}(T)+C_{e l}^{e p}(T), \\
& C_{e l}^{D}(T)=\frac{128}{K_{B} T^{2}} \sum_{k, k_{1}}\left|g_{k}\right|^{2}\left|D\left(k_{1}, k_{2}\right)\right|^{2} \\
& \times\left\{D\left(\widetilde{\epsilon}_{k_{1}}, \bar{\epsilon}_{q}\right) \epsilon_{k_{1}}\right. \\
& \times\left[\bar{N}\left(\epsilon_{k c}\right) n\left(\widetilde{\epsilon_{k_{1}}}\right)+N\left(\epsilon_{k c}\right) \widetilde{\epsilon_{k_{1}}} \widetilde{n}\left(\widetilde{\epsilon_{k_{1}}}\right)\right] \\
& +\widetilde{\epsilon}_{q c} D\left(\widetilde{\epsilon}_{q c}, \bar{\epsilon}_{q}\right) \\
& \left.\times\left[\epsilon_{k_{1}}\left(n_{k_{1}}^{2}-1\right) n\left(\widetilde{\epsilon_{q c}}\right)+2 n_{k_{1}} \bar{n}\left(\widetilde{\epsilon_{q c}}\right)\right]\right\}, \\
& =\frac{288}{K_{B} T^{2}} \sum_{k, k_{1}, k_{2}}\left|g_{k}\right|^{2}\left|V_{3}\left(k_{1}, k_{2},-k\right)\right|^{2} \\
& \times\left\{\eta_{1} N\left(\epsilon_{k c}\right) A^{( \pm \alpha)}+D_{s}^{(1)}\left(\widetilde{\epsilon}_{ \pm \alpha}, \widetilde{\epsilon}_{q}\right)\right. \\
& \left.+2 \widetilde{\epsilon_{q c}} D\left(\widetilde{\epsilon}_{q c}, \bar{\epsilon}_{q}\right)\left[n_{k_{1}} n_{k_{2}} \bar{n}\left(\widetilde{\epsilon}_{q c}\right)+n\left(\widetilde{\epsilon}_{q c}\right) n_{12}\right]\right\}, \\
& C_{e l}^{e p}(T)=\frac{4}{k_{B} T^{2}} \sum_{k}\left|g_{k}\right|^{2} \epsilon_{q c}^{2} \\
& \times\left\{D\left(\widetilde{\epsilon}_{k}, \widetilde{\epsilon}_{q}\right) \Omega\left(\bar{N}\left(\epsilon_{k c}\right) n\left(\widetilde{\epsilon}_{k}\right)+\widetilde{\epsilon}_{k} \widetilde{n}\left(\widetilde{\epsilon}_{k}\right) N\left(\epsilon_{k c}\right)\right)\right. \\
& +\left[\left(\frac{\epsilon_{k}^{3}\left(n_{k}^{2}-1\right)}{\epsilon_{q c}^{2}}+\widetilde{\epsilon}_{k}\left(\widetilde{n}_{k}^{2}-1\right)\left(\epsilon_{k} \epsilon_{q c}^{-1}+1\right)\right)\right. \\
& \left.\times n\left(\widetilde{\epsilon}_{q c}\right)+\widetilde{\Omega}\left(\widetilde{\epsilon}_{q c}\right) \bar{n}\left(\widetilde{\epsilon}_{q c}\right)\right] \\
& \left.\widetilde{\epsilon}_{q c} D\left(\widetilde{\epsilon}_{q c}, \bar{\epsilon}_{q}\right)\right\}
\end{aligned}
$$

The various symbols used in above expressions are defined as follows:

$$
\begin{aligned}
& N\left(\epsilon_{k c}\right)=\left(\frac{1}{2}\right)\left[N\left(3 \epsilon_{k_{F}}\right)+n\left(\epsilon_{c}\right)\right], \\
& N\left(x_{i}\right)=\left(e^{\beta x_{i}}+1\right)^{-1}, \\
& \widetilde{N}\left(\epsilon_{k_{i}}\right)=N\left(\epsilon_{k_{i}}\right)\left[1-N\left(\epsilon_{k_{i}}\right)\right] \\
& n\left(x_{i}\right)=\left(e^{\beta x_{i}}-1\right)^{-1}, \\
& \widetilde{n}\left(\epsilon_{k_{i}}\right)=n\left(\epsilon_{k_{i}}\right)\left[1+n\left(\epsilon_{k_{i}}\right)\right] \text {, } \\
& \eta_{i-1}=\frac{\epsilon_{k_{1}} \epsilon_{k_{2}} \cdots \epsilon_{k_{i}}}{\widetilde{\epsilon}_{k_{1}} \widetilde{\epsilon}_{k_{2}} \cdots \widetilde{\epsilon}_{k_{i}}}, \\
& S_{ \pm \alpha}=n_{k_{2}} \pm n_{k_{1}}, \\
& S_{ \pm \alpha}^{(1)}=\frac{\left[\left(n_{k_{2}}^{2}-1\right) \epsilon_{k_{2}} \pm\left(n_{k_{1}}^{2}-1\right) \epsilon_{k_{1}}\right]}{2}, \\
& A^{( \pm \alpha)}=S_{+\alpha} \widetilde{\epsilon_{+\alpha}} n(+\alpha) D\left(\tilde{\epsilon}_{+\alpha}, \bar{\epsilon}_{q}\right) \\
& \times\left[\bar{N}\left(\epsilon_{k c}\right)+N\left(\epsilon_{k c}\right) \epsilon_{+\alpha}\left(n\left(\epsilon_{+\alpha}\right)+1\right)\right] \\
& +S_{-\alpha} \widetilde{\epsilon_{+\alpha}} n(-\alpha) \times D\left(\tilde{\epsilon}_{+\alpha}, \bar{\epsilon}_{q}\right) \\
& \times\left[\bar{N}\left(\epsilon_{k c}\right)+N\left(\epsilon_{k c}\right) \epsilon_{-\alpha}\left(n\left(\epsilon_{-\alpha}\right)+1\right)\right], \\
& n_{12}=n_{k_{2}} \epsilon_{k_{1}}\left(n_{k_{1}}^{2}-1\right)+n_{k_{1}} \epsilon_{k_{2}}\left(n_{k_{2}}^{2}-1\right) \text {, } \\
& A_{\alpha}=S_{+\alpha} \bar{\epsilon}_{+\alpha} \delta\left(\epsilon^{2}-\widetilde{\epsilon}_{+\alpha}^{2}\right)+S_{-\alpha} \bar{\epsilon}_{-\alpha} \delta\left(\epsilon^{2}-\widetilde{\epsilon}_{-\alpha}^{2}\right) \text {, } \\
& D_{s}^{(1)}\left(\widetilde{\epsilon}_{ \pm \alpha}, \bar{\epsilon}_{q}\right)=S_{+\alpha}^{(1)} \epsilon_{+\alpha} n\left(\epsilon_{+\alpha}\right) D\left(\widetilde{\epsilon}_{+\alpha}, \bar{\epsilon}_{q}\right) \\
& +S_{-\alpha}^{(1)} \epsilon_{-\alpha} n\left(\epsilon_{-\alpha}\right) D\left(\widetilde{\epsilon}_{-\alpha}, \bar{\epsilon}_{q}\right), \\
& D_{s}\left(\widetilde{\epsilon_{ \pm \alpha}}, \bar{\epsilon}_{q}\right)=S_{+\alpha} \widetilde{\epsilon_{+\alpha}} n\left(\epsilon_{+\alpha}\right) D\left(\widetilde{\epsilon_{+\alpha}}, \bar{\epsilon}_{q}\right) \\
& +S_{-\alpha} \widetilde{\epsilon_{-\alpha}} n\left(\epsilon_{-\alpha}\right) D\left(\widetilde{\epsilon_{-\alpha}}, \bar{\epsilon}_{q}\right), \\
& \bar{N}\left(\epsilon_{k c}\right)=\left(\frac{1}{2}\right)\left[3 \epsilon_{k_{F}} \widetilde{N}\left(3 \epsilon_{k_{F}}\right)+\widetilde{\epsilon}_{c} \widetilde{n}\left(\epsilon_{c}\right)\right] \text {, }
\end{aligned}
$$




$$
\begin{aligned}
& n\left(\widetilde{\epsilon_{q c}}\right)=N\left(3 \widetilde{\epsilon}_{q}\right) n^{2}\left(\widetilde{\epsilon}_{q}^{c}\right) \\
& \times\left[\widetilde{n}\left(\widetilde{\epsilon}_{q}^{c}\right)-n\left(\widetilde{\epsilon}_{q}^{c}\right) N\left(3 \tilde{\epsilon}_{q}\right)\right]^{-1}, \\
& \bar{n}\left(\widetilde{\epsilon}_{q c}\right)=\widetilde{\epsilon}_{q c} \widetilde{N}\left(3 \widetilde{\epsilon}_{q}\right) \widetilde{n}\left(\widetilde{\epsilon}_{q}^{c}\right) n^{2}\left(\widetilde{\epsilon}_{q}^{c}\right) \\
& \times\left[\widetilde{n}\left(\widetilde{\epsilon}_{q}^{c}\right)-n\left(\widetilde{\epsilon}_{q}^{c}\right) N\left(3 \widetilde{\epsilon}_{q}\right)\right]^{-2}, \\
& D\left(\widetilde{\epsilon}_{i}, \bar{\epsilon}_{q}\right)=\left(\widetilde{\epsilon}_{i}^{2}-\bar{\epsilon}_{q}^{2}\right)^{-2} \text {, } \\
& \widetilde{\epsilon_{ \pm \alpha}}=\widetilde{\epsilon_{k_{2}}} \pm \widetilde{\epsilon_{k_{1}}}, \quad \widetilde{\epsilon_{ \pm \beta}}=\widetilde{\epsilon_{k_{1}}} \pm \widetilde{\epsilon_{k_{2}}} \pm \widetilde{\epsilon_{k_{3}}}, \\
& n_{k_{i}}=\operatorname{coth}\left(\frac{\beta \epsilon_{k_{i}}}{2}\right) \text {, } \\
& \widetilde{n_{k_{i}}}=\frac{\widetilde{\epsilon_{k_{i}}}}{\epsilon_{k_{i}}} \operatorname{coth}\left(\frac{\beta \epsilon_{k_{i}}}{2}\right) \text {, } \\
& \Omega=\left(\frac{-8 \widetilde{\epsilon}_{k}^{2}}{\epsilon_{k}}+\frac{2 \epsilon_{k}^{3}}{\epsilon_{q c}^{2}}\right), \\
& \widetilde{\Omega}=\left(\frac{\epsilon_{k}^{2}}{\epsilon_{q c}^{2}}+\frac{4 \epsilon_{k} \widetilde{n}_{k}}{\epsilon_{q c}}+\widetilde{n_{k}}\right) \text {, } \\
& \epsilon_{q c}=3 \epsilon_{q}+\epsilon_{q}^{c} \text {. }
\end{aligned}
$$

\section{Discussion and Conclusions}

Above investigations obviously exhibit that the EDOS not only depends on electron energy but also becomes a function of various renormalized/perturbed mode energies, pairon energies, temperature, anharmonicity, and defect concentration. Based on this model, the electron phonon contribution to EDOS has been depicted in Figure 1. This work investigates the general theory of EHC for HTS and reveals that the EHC is not a simple quantity as $(\sim \gamma T)$ but comprises of defect contribution $C_{e l}^{D}(T)$, anharmonic contribution $C_{e l}^{3 A}(T)$, and electron-phonon contribution $C_{e l}^{e p}(T)$ through electron-phonon coupling constant $\mathrm{g}(\mathrm{k})$. A careful examination of these terms infers that $C_{e l}^{D}(T)$ varies with temperature as $\sim T^{-2}$ along with defect concentration, pairon distribution functions $\bar{N}\left(\epsilon_{k c}\right), \bar{n}\left(\widetilde{\epsilon}_{q c}\right)$, phonon distribution, and electron, phonon, and pairon frequencies, which ensures high sensitivity at low-temperatures. The anharmonic contribution depends on the nature of anharmonic forces as well as on various temperature dependent terms. The contribution to EHC by electron-phonon interaction is heavily influenced by pairon distribution functions, renormalized phonon distribution functions with sophisticated variation of electron, phonon, and pairon frequencies in fundamental, renormalized, and perturbed modes.

It emerges from the present study that the present formulation is capable to explain the EHC with the signatures of electron-phonon interaction in HTS along with their normal phase. This theory can be applied to model calculations of HTS.

\section{Acknowledgments}

The authors (A. Singh and H. Singh) are thankful to CSIR and MHRD, New Delhi, India, for the financial support to carry out this research work.

\section{References}

[1] M. Kulic, "Interplay of electron-phonon interaction and strong correlations: the possible way to high-temperature superconductivity," Physics Reports, vol. 338, no. 1-2, pp. 1-264, 2000.

[2] C. K. Poole, H. A. Farach, and R. J. Creswick, Handbook of Superconductivity, Edited by J. R. C. K. Poole, Academic Press, New York, NY, USA, 2000.

[3] R. A. Fisher, J. E. Gordan, and N. E. Phillips, Handbook of High Temperature Superconductivity: Theory and Experiment, Edited by J. R. Schrieffer and J. S. Brooks, Springer, New York, NY, USA, 2007.

[4] E. G. Maksimov, A. E. Karakozov, B. P. Gorshunov, E. S. Zhukova, G. Ponomarev, and M. P. N. Dressel, "Electronic specific heat of two-band layered superconductors: analysis within the generalized two-band $\alpha$ model," Physical Review B, vol. 84, Article ID 174504, 8 pages, 2011.

[5] J. W. Loram, K. A. Mirza, J. R. Cooper, and W. Y. Liang, "Electronic specific heat of $\mathrm{YBa}_{2} \mathrm{Cu}_{3} \mathrm{O}_{6+x}$ from 1.8 to $300 \mathrm{~K}$," Physical Review Letters, vol. 71, no. 11, pp. 1740-1734, 1993.

[6] B. D. Indu, "Theory of lattice specific heat of an isotopically disordered anharmonic crystal," International Journal of Modern Physics B, vol. 4, article 1379, 1990.

[7] B. D. Indu, "Enhanced phonon density of states in impure anharmonic crystals," Modern Physics Letters B, vol. 6, pp. 1665-1672, 1992.

[8] K. N. Pathak, "Theory of anharmonic crystals," Physical Review A, vol. 139, pp. 1569-1580, 1965.

[9] P. K. Sharma and R. Bahadur, "Thermal conductivity for phonon scattering by substitutional defects in crystals," Physical Review B, vol. 12, no. 4, pp. 1522-1530, 1975.

[10] D. N. Zubarev, "Double-time Green functions in statistical physics," Soviet Physics. Uspekhi, vol. 3, pp. 320-345, 1960.

[11] V. Ashokan, B. D. Indu, and A. K. Dimri, "Signature of electronphonon interaction in high temperature superconductors," AIP Advances, vol. 1, Article ID 032101, 16 pages, 2011. 

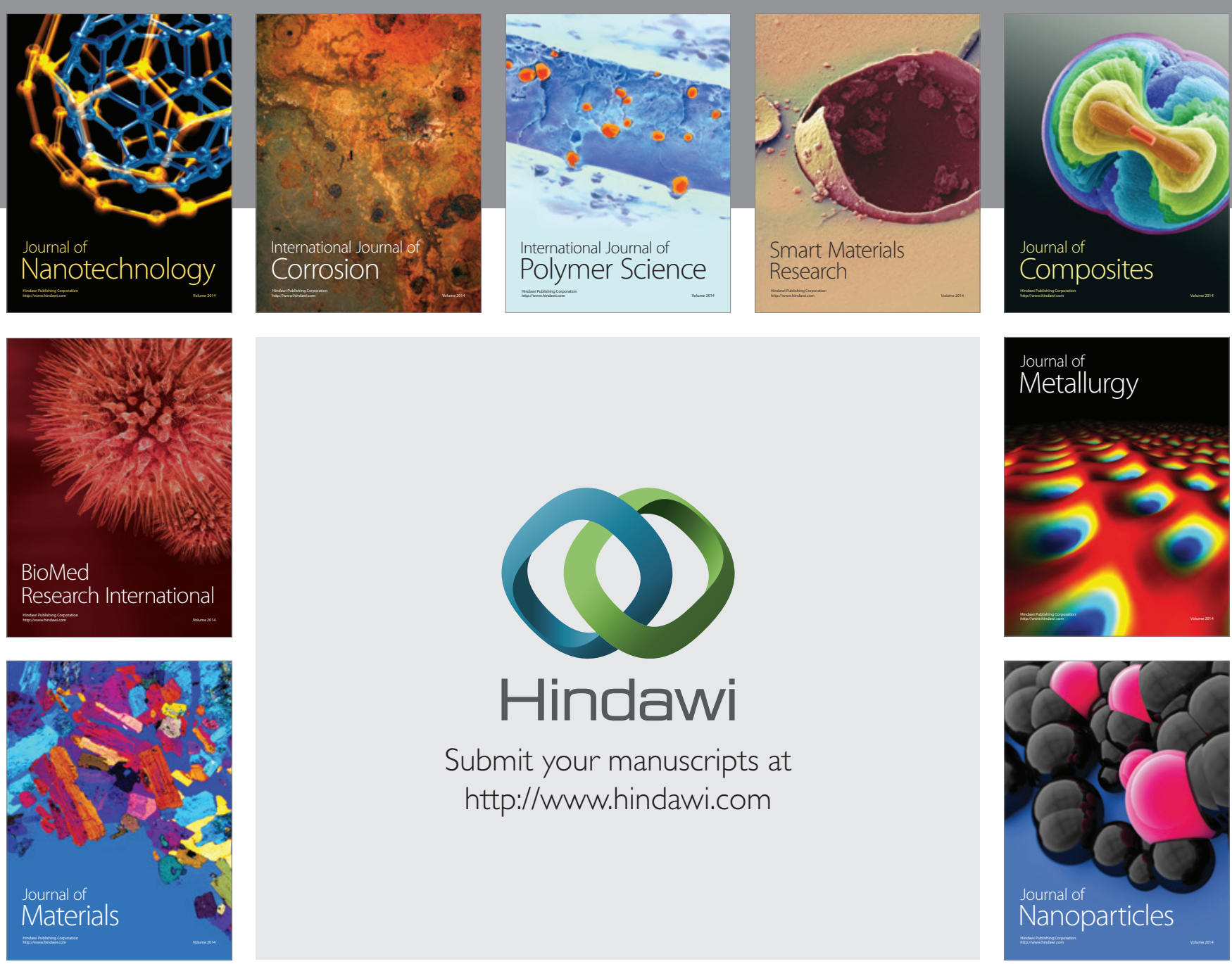

Submit your manuscripts at http://www.hindawi.com
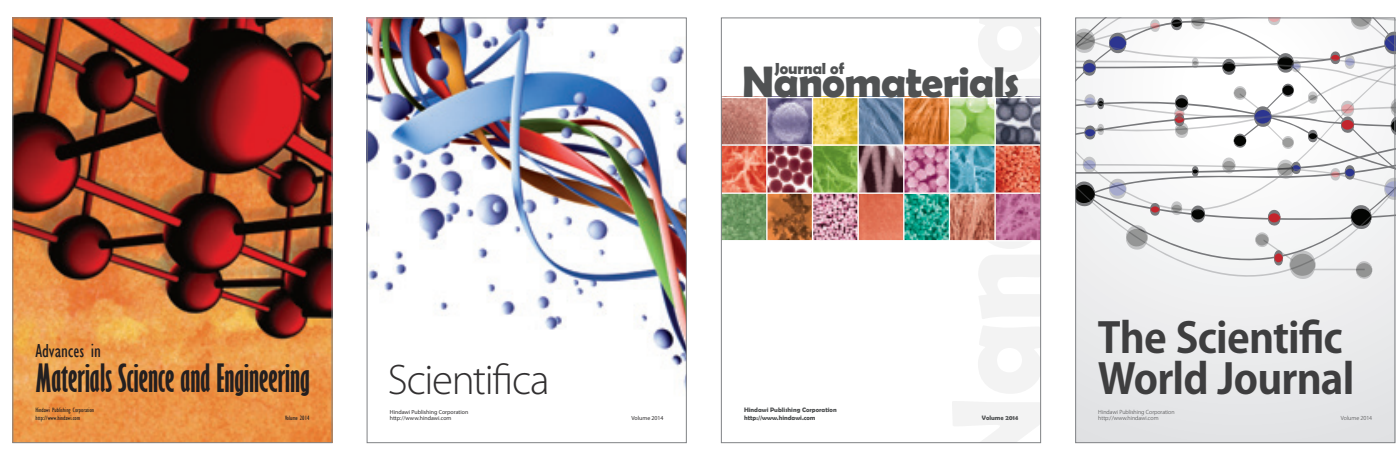

\section{The Scientific World Journal}
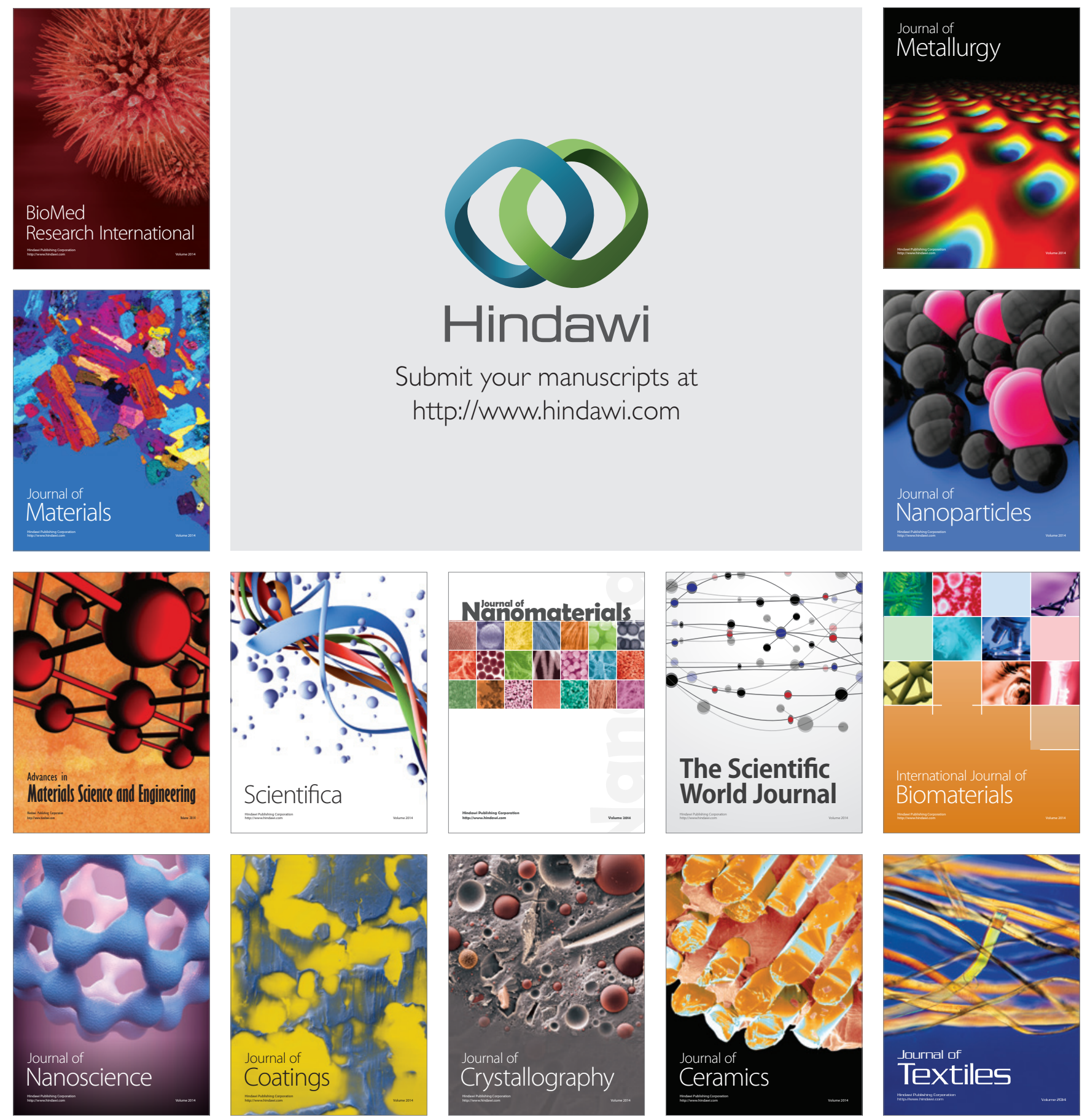